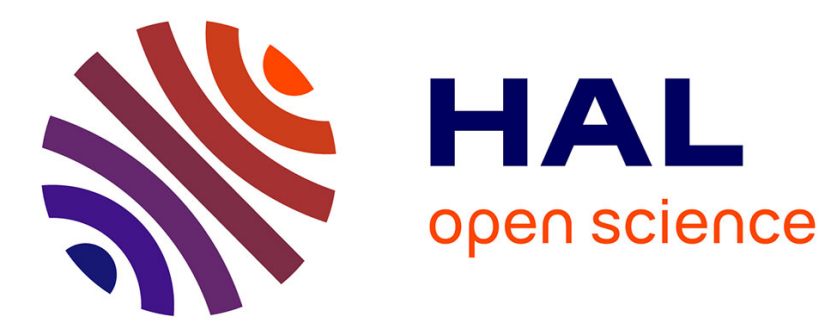

\title{
Sur l'élaboration de schémas nucléaires complexes par ordinateur
}

\author{
B. Escudié, A. Gerson, A. Charvet, R. Chéry
}

\section{To cite this version:}

B. Escudié, A. Gerson, A. Charvet, R. Chéry. Sur l'élaboration de schémas nucléaires complexes par ordinateur. Revue de Physique Appliquée, 1969, 4 (2), pp.123-124. 10.1051/rphysap:0196900402012300 . jpa-00243174

\section{HAL Id: jpa-00243174 https://hal.science/jpa-00243174}

Submitted on 1 Jan 1969

HAL is a multi-disciplinary open access archive for the deposit and dissemination of scientific research documents, whether they are published or not. The documents may come from teaching and research institutions in France or abroad, or from public or private research centers.
L'archive ouverte pluridisciplinaire HAL, est destinée au dépôt et à la diffusion de documents scientifiques de niveau recherche, publiés ou non, émanant des établissements d'enseignement et de recherche français ou étrangers, des laboratoires publics ou privés. 


\title{
SUR L'ÉLABORATION DE SGHÉMAS NUGLÉAIRES COMPLEXES PAR ORDINATEUR
}

\author{
B. ESGUdiÉ, A. GERSON, A. CHARVET et R. GHÉRY, \\ Institut de Physique Nucléaire, 69-Lyon.
}

\begin{abstract}
Résumé. - La règle de Ritz appliquée au traitement sur ordinateur fournit un moyen commode de traitement de l'information des spectres $\beta$ ou $\gamma$. Nous proposons ici un groupe de programmes adapté à cette analyse.

Abstract. - Using Ritz's principle in a numerical manner produces easy information processing in the study of nuclear $\beta-\gamma$ decay schemes. We use three convenient programmes adapted for this analysis.
\end{abstract}

L'emploi systématique de la règle des sommes (règle de Ritz) adaptée au traitement automatique (ordinateur) fournit un procédé rapide de tri de l'information contenue dans les spectres complexes $(\beta$ ou $\gamma$ ). Dans un premier temps, le programme RS1 donne, à partir d'une liste de raies $\left(E_{i}\right)$, toutes les possibilités $E_{i}+E_{j}=E_{k} \pm \Delta E, \quad E_{i} \leqslant E_{j}$, classées par $E_{k}$ décroissantes. $\Delta E$ est choisi à partir de la précision des mesures des $E_{i}$. Les programmes RS2a et $\mathrm{RS} 2 \mathrm{~b}$ calculent ensuite, par la règle de Ritz, tous les niveaux $E_{k}$ et $E_{k^{\prime}}$ s'excluant l'un l'autre, à partir d'une liste de niveaux $E_{i}$ supposés connus ( $f g .1$ et 2 ).

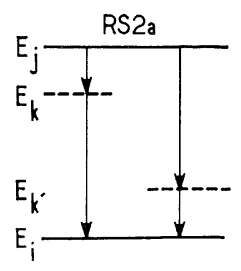

Fig. 1.

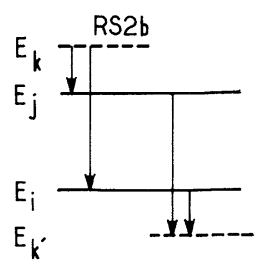

FIG. 2.
Les programmes RS2a et RS2b fournissent les niveaux $E_{k} E_{k^{\prime}}$ à partir de la liste des niveaux $E_{i} E_{j}$ supposés connus.

Chaque niveau $E_{k}$ est affecté d'un indice de communauté $I(k)$, égal à son nombre d'apparitions au cours du calcul. Un indice $I(k)$ assez élevé indique une probabilité d'existence réelle du niveau $E_{k}$ assez grande. On utilisera le programme RS2a lorsqu'on connaîtra des niveaux d'énergie moyenne pour rechercher des niveaux non supérieurs. Le programme RS2b essaie de déterminer des niveaux d'énergie supérieure aux énergies des niveaux connus. Il peut s'employer, par exemple, quand on connaît les premières « bandes » et qu'on les a confirmées par la mesure. La comparaison des indices de communauté $I(k)$ et $I\left(k^{\prime}\right)$ permet généralement d'ajouter une liste de nouveaux niveaux à la liste $E_{i}$. Cette nouvelle liste fera l'objet d'un traitement décrit plus loin.

Le programme RS3 propose, à partir d'un seul niveau maximal, un ensemble de schémas possibles de désexcitation de ce niveau vers le fondamental. A partir du niveau $E_{M}$, on construit les niveaux $E_{k}$ et $E_{k^{\prime}}$ exclusifs. Les transitions fournies par ces niveaux conduisent,

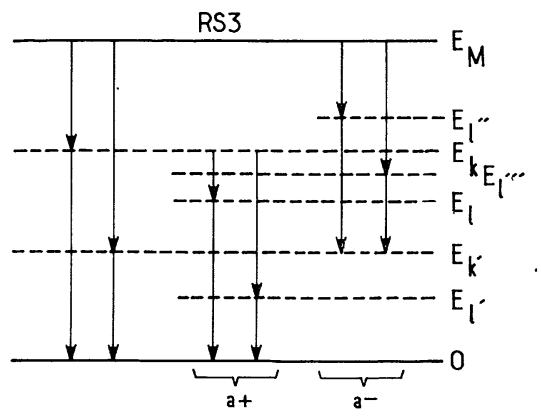

FIG. 3. - Le programme RS3

fournit les voies de désexcitation possibles d'un niveau $E_{M}$.

par le même traitement, aux nouvelles possibilités $E_{l}, E_{l^{\prime}}, E_{l^{\prime \prime}}, E_{l^{\prime \prime \prime}}\left(\right.$ fig. 3 ). Les cascades $a^{+}$et $a^{-}$s'excluent $^{\prime}$ mutuellement. Le programme RS3 effectue une sélection optimale des schémas partiels, obtenus par adjonc- 
tion des cascades (a, b, c, d...). Le critère d'optimalisation repose sur l'analyse marginale des indices de communauté des niveaux obtenus.

L'ordre d'emploi de ces trois programmes reflète le procédé d'obtention des niveaux. RS2a et RS2b permettent, à partir de niveaux connus, d'en identifier d'autres. Ceux-ci étant supposés certains fournissent un certain nombre de schémas partiels de désexcitation par l'intermédiaire de RS3. Le choix entre plusieurs niveaux possibles dépend directement de leur indice $I$ respectif. Dans une paire de niveaux exclusifs, un examen comparatif des indices permet en général de décider de la validité d'un niveau fourni.

En conclusion, on peut attendre de cet ensemble une simplification de l'étude d'un schéma par choix et tri correct de l'information comme le confirme l'application faite à l'élaboration du schéma nucléaire de ${ }^{168} \mathrm{Yb}$.

Un rapport contenant les programmes et la méthode de leur emploi est disponible auprès des auteurs. 